\title{
Metabolic alterations due to IDH1 mutation in glioma: opening for therapeutic opportunities?
}

\author{
Dana A N Mustafa ${ }^{*}$, Sigrid M Swagemakers ${ }^{2}$, Laura Buise ${ }^{1}$, Peter J van der Spek ${ }^{2}$ and Johan M Kros ${ }^{1}$
}

Recently heterozygous mutations in the active site of the enzyme isocitrate dehydrogenase 1 (IDH1) were discovered in glioblastomas [1]. In cohorts of glioma patients the IDH1 mutation appeared to be a strong predictor of clinical outcome, overruling histological malignancy grade [2]. IDH1 is an enzyme of the tricarboxylic acid (TCA) cycle and is located in the cytosol, where it produces NADPH by transforming isocitrate into $\alpha$-ketoglutarate. Because the mutant enzyme displays neomorphic activity through NADPH-dependent transformation of $\alpha$-ketoglutarate into 2-hydroxyglutarate $(2 \mathrm{HG})$, the tumorigenic role of the increased levels of $2 \mathrm{HG}$ has become a target of speculation [3]. IDH1 mutation alters the cellular metabolism and epigenetic phenotype influencing cellular proliferation. IDHI mutation infers increased levels of $D 2 H G D H$ leading to the inhibition of DNA and histone demethylating enzymes, resulting in the glioma-CpG island phenotype [4]. Altered concentrations of pyruvate kinase M2 play also a role in histone modifications which are associated with the transcription of the proliferation-related cyclin D1 and c-MYC [5]. In addition, IDH1 mutant cells show alterations in glutamine, fatty acid and citrate synthesis pathways, which all may have their influence on cellular proliferation [5].

The changes in $I D H 1$ function affect the glucose metabolism, which may explain the different biological behaviour of tumors with and without the IDH1 mutation [6]. In order to detect these changes, we compared the expression levels of the genes participating in the TCA cycle and in the anaerobic glycolysis in $33 \mathrm{IDH} 1$ mutated samples (3 astrocytomas WHO grade II; 6 astrocytomas WHO grade III; 4 glioblastomas; 9 oligodendrogliomas WHO grade II; 11 oligodendrogliomas WHO grade III) and in 39 IDH1 wild-type glioma samples (10 astrocytomas WHO grade III; 26 glioblastomas; 1 oligodendroglioma WHO grade II; 2 oligodendroglioma WHO grade III) and in four samples

\footnotetext{
* Correspondence: d.mustafa@erasmusmc.nl

'Department of Pathology, Erasmus Medical Center, Dr. Molewaterplein 50, 3000 CA, Rotterdam, The Netherlands

Full list of author information is available at the end of the article
}

of normal brain (Table 1). We found expressional differences of 16/24 genes (Figure 1). The IDH1 mutated cells seem to compensate for the low production of $\alpha$ ketoglutarate by overexpressing $D 2 H G D H$ and $L 2 H G D H$ in the cytoplasm. They also overexpress GLUD1, which converts glutamate to $\alpha$-ketoglutarate inside the mitochondria. In addition, we found that $I D H 1$ mutated cells overexpress HIF1AN. The HIF1AN gene inhibits HIF1 $\alpha$. Since HIFl $\alpha$ acts as an oxygen sensor that promotes angiogenesis, the formation of dysfunctional tumor vasculature is counteracted in IDH1 mutated cells. Furthermore, the $I D H 1$ mutated cells overexpress the $L D H B$ gene while cells without $I D H 1$ mutation overexpress the $L D H A$ gene. The present results illustrate that tumor cells without IDH1 mutation switch their energy production from a low rate of glycolysis followed by the TCA cycle to a high rate of glycolysis followed by aerobic glycolysis ( $L D H A$ up-regulation; Figure 1). The resulting lactate acid production causes tissue acidosis known as the Warburg effect. In invasive cancers, the $\mathrm{pH}$ of the extracellular space increases the infiltrative potential of the tumor cells [7]. In addition, normalization of the extracellular $\mathrm{pH}$ by alterations of the enzymatic actions of $L D H A$ and $L D H B$ influences the progression of cancer cells [8,9]. It may well be that glial tumor cells with $I D H 1$ mutation tend to correct their energy production through the TCA cycle by overexpressing $L D H B$ (Figure 1). By doing so, they normalize the tissue $\mathrm{pH}$ which offers yet another explanation for the less

\begin{tabular}{lll}
\multicolumn{3}{l}{ Table 1 Percentages of glioma types and grades } \\
\hline WHO grades & IDH1 mutation & IDH1 wild type \\
\hline A II & $4 \%$ & $0 \%$ \\
A III & $8 \%$ & $14 \%$ \\
GBM & $6 \%$ & $36 \%$ \\
O II & $13 \%$ & $1 \%$ \\
O III & $15 \%$ & $3 \%$ \\
\hline
\end{tabular}

Legends: A II low-grade astrocytoma (WHO grade 2), A III anaplastic astrocytoma (WHO grade 3), GBM glioblastoma (WHO grade 4), O II low-grade oligodendroglioma (WHO grade 2), O III anaplastic oligodendroglioma (WHO grade 3). 


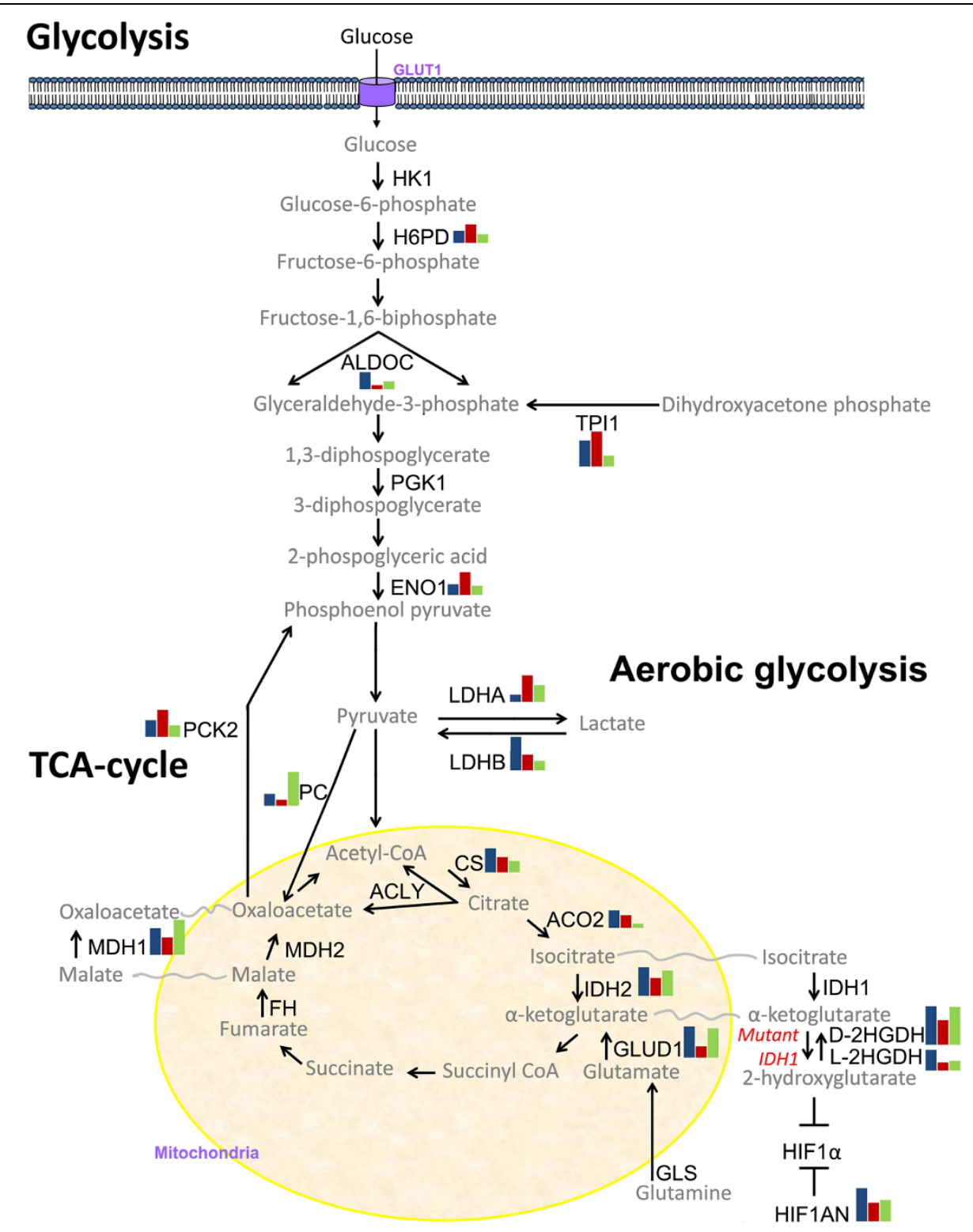

Figure 1 Overview of alterations in glucose metabolism in the $I D H 1$ mutated and $I D H 1$ wild-type gliomas relative to glucose

metabolism in normal brain. The enzymes included in the analysis are shown in black. The bar diagrams show the averaged expressional levels of genes that were significantly differently expressed in the IDH1 mutated gliomas ( $n=33$, blue bars on the left); IDH1 wild-type ( $n=39$, red bars in the middle); and normal brain ( $n=4$, green bars on the right). Glycolysis leads to the production of pyruvate. In normal brain, pyruvate is converted to acetyl-CoA, which enters the TCA cycle in the mitochondria. But in the IDH1 wild-type tumors, pyruvate is metabolized to lactate ("the Warburg effect" in which the aerobic glycolysis serves as a quick energy source that results in tissue acidosis). To ensure the origin and quality of the tissues, all tissues were assessed by a qualified pathologist before isolation. IDH1 status was checked using IHC and the standard PCR test. Total RNA was isolated with the RNA-Bee (Campro, Veenendaal, The Netherlands). cDNA was prepared using the RevertAid H Minus First Strand cDNA synthesis kit (Fermentas, St Leon-Rot, Germany). The resulting CDNA preparations were analysed by real-time PCR with SYPR green master mix solution (Applied Biosystems, Nieuwerkerk a/d IJssel, The Netherlands). PCRs were performed in a $25 \mu \mathrm{L}$ reaction volume in an Applied BioSystems 7900HT Fast Real-Time PCR system. Negative controls included minus RT and $\mathrm{H}_{2} \mathrm{O}$-only samples, which were negative in all cases. Expression of B2M, HPRT1 and PBGD was used as a reference to control sample loading and RNA quality. Differences in mRNA concentrations were determined using the $t$-test, with $P<0.01$ being considered statistically significant. All statistical tests were two-sided.

aggressive biological behavior of the IDH1 mutated gliomas.

We conclude that gliomas with IDH1 mutation normalize their glucose metabolism, which appears to result in a slower tumor progression. Depending on the $I D H 1$ status of the tumor, specific interference with the glucose metabolism and aerobic glycolysis should therefore be considered for future therapeutic strategies. 


\section{Competing interests}

All authors declare that they have no competing interests.

\section{Authors' contributions}

DM and LB carried out the molecular genetic analyses; SS and PvdS carried out the data analysis and DM and JMK conceptualized and designed the study and wrote the manuscript. All authors read and approved the final manuscript.

\section{Acknowledgements}

The authors thank Mr. M. van der Weiden for his technical assistance and Mr. F. van der Panne for assistance with the photography.

\section{Author details}

'Department of Pathology, Erasmus Medical Center, Dr. Molewaterplein 50, 3000 CA, Rotterdam, The Netherlands. ${ }^{2}$ Departments of Bio-Informatics, Erasmus Medical Center, Dr. Molewaterplein 50, 3015 GE, Rotterdam, The Netherlands.

Received: 25 November 2013 Accepted: 2 January 2014

Published: 9 January 2014

\section{References}

1. Parsons DW, Jones S, Zhang X, Lin JC, Leary RJ, Angenendt P, et al: An integrated genomic analysis of human glioblastoma multiforme. Science 2008, 321:1807-1812.

2. Yan $H$, Parsons DW, Jin G, McLendon R, Rasheed BA, Yuan W, et al: IDH1 and IDH2 mutations in gliomas. N Engl J Med 2009, 360:765-773.

3. Dang L, White DW, Gross S, Bennett BD, Bittinger MA, Driggers EM, et al: Cancer-associated IDH1 mutations produce 2-hydroxyglutarate. Nature 2009, 462:739-744.

4. Borodovsky A, Seltzer MJ, Riggins GJ: Altered cancer cell metabolism in gliomas with mutant IDH1 or IDH2. Curr Opin Oncol 2012, 24:83-89.

5. Venneti $\mathrm{S}$, Thompson CB: Metabolic modulation of epigenetics in gliomas. Brain Pathol 2013, 23:217-221.

6. Reitman $\mathrm{ZJ}$, Yan H: Isocitrate dehydrogenase 1 and 2 mutations in cancer: alterations at a crossroads of cellular metabolism. J Natl Cancer Inst 2010, 102:932-941.

7. Mirebeau-Prunier D, Le Pennec S, Jacques C, Fontaine JF, Gueguen N, Boutet-Bouzamondo N, et al: Estrogen-related receptor alpha modulates lactate dehydrogenase activity in thyroid tumors. PLOS One 2013, 8:e58683.

8. Ho J, de Moura MB, Lin Y, Vincent G, Thorne S, Duncan LM, et al: Importance of glycolysis and oxidative phosphorylation in advanced melanoma. Mol Cancer 2012, 11:76.

9. McCleland ML, Adler AS, Deming L, Cosino E, Lee L, Blackwood EM, et al: Lactate dehydrogenase $B$ is required for the growth of KRAS-dependent lung adenocarcinomas. Clin Cancer Res 2013, 19:773-784.

doi:10.1186/2051-5960-2-6

Cite this article as: Mustafa et al:: Metabolic alterations due to IDH1 mutation in glioma: opening for therapeutic opportunities? Acta

Neuropathologica Communications 2014 2:6.

\section{Submit your next manuscript to BioMed Central and take full advantage of:}

- Convenient online submission

- Thorough peer review

- No space constraints or color figure charges

- Immediate publication on acceptance

- Inclusion in PubMed, CAS, Scopus and Google Scholar

- Research which is freely available for redistribution 